\title{
Changes in cyclic GMP level and phosphodiesterase activity during follicular development in the rat ovary
}

\author{
C.Q. Fu ${ }^{1,2}$, F.X. Shi' ${ }^{2}$ Z.H. Zhang ${ }^{3}$, J.R. Li ${ }^{1,2}$, X.H. Huang ${ }^{3}$ and Z.C. Wang ${ }^{2,3}$ \\ ${ }^{1}$ Jinhua Polytechnic College, Jinhua, China \\ ${ }^{2}$ College of Animal Science and Technology, \\ Nanjing Agricultural University, Nanjing, China \\ ${ }^{3}$ Provincial Key Laboratory for Developmental Biology and Neurosciences, \\ College of Life Sciences, Fujian Normal University, Fuzhou, China \\ Corresponding author: Z.C. Wang \\ E-mail: zcwang@fjnu.edu.cn
}

Genet. Mol. Res. 13 (3): 5919-5928 (2014)

Received May 22, 2013

Accepted March 13, 2014

Published August 7, 2014

DOI http://dx.doi.org/10.4238/2014.August.7.7

\begin{abstract}
Guanosine 3',5'-cyclic monophosphate (cGMP), as a second messenger, plays potential roles in ovarian functions. To elucidate the role of phosphodiesterase (PDE) in cGMP signaling during ovarian follicular development, the present study was conducted to investigate ovarian cGMP level and cGMP-PDE activity by radioimmunoassay (RIA) in postnatal rats, immature rats during gonadotropin-primed follicular development, ovulation and luteinization, adult rats during normal estrous cycle, and aged rats that spontaneously developed persistent estrus (PE). All four rat models were confirmed by histological examination of one ovary, and the other ovary was used for RIA. In postnatal rats, cGMP level was high at birth and decreased dramatically by Day 5, and then, it increased maximally at Day 10 and declined at Day 21. However, cGMP-PDE activity did not significantly change during Days 1 to 10 , but increased significantly on Day 21. In immature female rats, cGMP level markedly
\end{abstract}


decreased upon treatment with equine chorionic gonadotropin (eCG), while cGMP-PDE activity did not show any significant changes; however, ovarian cGMP level and cGMP-PDE activity increased after injection of an ovulatory dose of human chorionic gonadotropin (hCG) for induction of ovulation and luteinization. In adult rats during normal estrous cycle, cGMP level was high on proestrus and metestrus days, while cGMP-PDE activity was high on estrus day. In PE rats, ovarian cGMP level was similar to that in adult rats on estrus and diestrus days but lower than that on proestrus and metestrus days; ovarian cGMP-PDE activity was lower than that on estrus days but similar as the other estrous cycle days. In addition, there was a significant negative correlation between ovarian cGMP level and cGMP-PDE activity during normal estrous cycles in the adult rat $(\mathrm{r}=-0.7715, \mathrm{~N}$ $=16, \mathrm{P}<0.05)$, but not in the postnatal rat $(\mathrm{r}=-0.1055, \mathrm{~N}=20, \mathrm{P}>$ 0.05). Together, the results of our present study indicated that ovarian cGMP levels were not dependent on cGMP-PDE activity during early postnatal development, but highly dependent on cGMP-PDE activity in the adult rat. This implies that mechanisms of cGMP signaling involved in ovarian functions are stage-specific in the rat.

Key words: Cyclic GMP; Granulosa cell; Ovary; Phosphodiesterase; Rat

\section{INTRODUCTION}

In mammals, the production of a viable oocyte is dependent on the critical influences of gonadotropins $(\mathrm{FSH} / \mathrm{LH})$ on ovarian functions. On the other hand, the actions of FSH and LH on ovarian functions are mediated in large part through increased production of the second messenger cyclic AMP (cAMP) and subsequent activation of downstream signaling pathways (Richards et al., 1995). Although the importance of cAMP as a second messenger influencing the ovary is well recognized (Aharoni et al., 1995; Das et al., 1996; Chaube et al., 2006; Tatsukawa et al., 2006; Wang et al., 2007), other hormones and factors acting through diverse signaling pathways also modulate ovarian functions. In this regard, a large body of evidence indicates the potential roles of cyclic GMP (cGMP) as another second messenger mediating a wide range of influences on the ovary (Sirotkin et al., 2000; LaPolt et al., 2003).

The concentration of $\mathrm{cAMP} / \mathrm{cGMP}$ is determined not only by the activation of cyclases but also by the expression and activity of PDEs. The synthesis of cGMP is accomplished by two distinct classes of guanylyl cyclases, particulate and soluble, both of which now appear to have relevance to ovarian physiology (Shi et al., 2004; Davies, 2006). The only known route for breakdown of cyclic nucleotides in the cell is through PDEs (Wang, 2006; Wang and Shi, 2007). However, such studies on cAMP-specific PDEs (cAMP-PDEs) are limited (Wang and Shi, 2007). Recent work in several cells and tissues suggests that enzymes regulating the breakdown of cGMP, as opposed to its synthesis, are pivotal in maintaining the role of cGMP in cellular functions (Murthy, 2001; Broderick et al., 2003; Mullershausen et al., 2003). Therefore, it is clear that as for other components of cGMP-dependent signaling, further work is required to understand the role of cGMP-PDE in ovarian functions. 
To date, however, little information is available regarding the regulated expression and actions of cGMP-PDE in the ovary (Piriev et al., 2003). Given the growing importance of cGMP as a second messenger in the ovary and the minimal information regarding the control of cGMP concentrations in gonadal tissues, our current study utilized different rat models to examine the roles of cGMP-PDE in ovarian functions.

\section{MATERIAL AND METHODS}

\section{Animals and experimental design}

Sprague-Dawley rats (Wushi Experimental Animal Supply Center, Fuzhou, China) were used. Animals were maintained under a 14-h light (5 am-7 pm), 10-h dark schedule with food and water available ad libitum. The experimental protocol was approved and in accordance with the Guide for the Care and Use of Laboratory Animals prepared by the Institutional Animal Care and Use Committee, Nanjing Agricultural University and Fujian Normal University.

The experimental design was exactly similar to our previous study (Wang et al., 2007). Four rat models, postnatal rat model, gonadotropin-primed immature rat model, adult rat model and aged rat model were used, where each treatment group consisted of 4 animals. Briefly, neonatal rats at $1,5,7,10$, and 21 days of age (the day of birth was assumed as Day 1) were used for the postnatal rat model; immature rats (25-26 days old) received a subcutaneous injection of equine chorionic gonadotropin (eCG, Sigma Chemical Co., St. Louis, MO, USA; $10 \mathrm{IU}$ ) at $10 \mathrm{am}$, followed $52 \mathrm{~h}$ later by an ovulatory dose (30 IU) of human chorionic gonadotropin (hCG, Sigma Chemical Co., St. Louis, MO, USA) and used for the immature rat model; adult rats during normal estrous cycles were used for the adult rat model; and aged rats with persistent estrous (PE) induced spontaneously with at least 15 consecutive days of vaginal cornification were used for the aged rat model.

Ovaries of immature and adult rat models were obtained at approximately $10 \mathrm{am}$. One ovary of each rat was fixed in $4 \%$ paraformaldehyde for histological evaluation, while the other ovary was snap-frozen and used for protein extraction and subsequent cGMP and phosphodiesterase assays.

\section{Radioimmunoassay of cGMP}

Levels of cGMP were determined by competition binding with [ $\left.{ }^{125} \mathrm{I}\right]$-succinyl guanosine 3', 5'-cyclic monophosphate tyrosyl methyl ester (ScGMP-TME) (Theilig et al., 2001). The amount of bound radioactivity was determined by gamma counting. The cGMP level of each sample was measured using commercial RIA kits (Shanghai University of Traditional Chinese Medicine, Shanghai, China). The minimum detection limits for cGMP was $0.1 \mathrm{nM}$ for nonacetylated samples; cross-reaction with cAMP was less than $0.001 \%$. The intra-coefficients of variation for cGMP were less than $6 \%$.

\section{cGMP-PDE activity assay}

cGMP-PDE activity was measured using $0.1 \mathrm{mM}$ cGMP as substrate, according to the method of Thompson and Appleman and as previously detailed (Sette and Conti, 1996; 
Nichols and Morimoto, 2000). Samples were assayed in a $200-\mu \mathrm{L}$ reaction mixture including $50 \mathrm{mM}$ Tris- $\mathrm{HCl}, \mathrm{pH} 8.0,150 \mathrm{mM} \mathrm{NaCl}$, and $0.1 \mathrm{mM}$ cGMP. After incubation at $34^{\circ} \mathrm{C}$ for 30 min, the reaction was terminated by adding an equal volume of $40 \mathrm{mM}$ Tris- $\mathrm{HCl}, \mathrm{pH} 7.5$, containing $10 \mathrm{mM}$ EDTA, followed by heat denaturation for exactly $1 \mathrm{~min}$ at $100^{\circ} \mathrm{C}$. cGMP level was measured by RIA. In general, the PDE assay consisted of measuring cGMP degradation during a timed incubation. PDE activity was expressed as the amount of cGMP hydrolyzed by PDE in the sample. Blank (background) control reactions were conducted using tissue samples that had been incubated in a boiling water bath for $5 \mathrm{~min}$. Protein concentration in the samples was measured according to the Bradford method (Bradford, 1976).

\section{Statistical analyses}

The results are expressed as the mean \pm SEM. Differences between treatments were evaluated by one-way or two-way analysis of variance, followed by Scheffe's post hoc test. P $<0.05$ was considered statistically significant. A paired or unpaired two-tailed Student $t$-test was used to detect significance between two series of data.

\section{RESULTS}

In all four rat models, one ovary of each experimental animal was used for histological examination, and the other ovary was homogenized for RIA of cGMP level and cGMP-PDE activity.

\subsection{Histological examination}

Our morphological examinations of the neonatal rat ovary were identical to those in our previous study (Wang et al., 2007). Ovaries of immature rats primed by gonadotropin and adult rats during normal estrous cycles showed typical histological changes (Figure 1). Before injection of gonadotropin, a 25-day-old ovary contained different kinds of follicles at different developmental stages, such as primordial follicles $(\mathrm{PF})$, primary follicles $(\mathrm{pF})$, antral follicles $(\mathrm{AF})$, and atresic follicles (DF), except for corpora lutea (CL) (Figure 1A). After injection of eCG, ovarian follicles began to develop, and some preovulatory follicles (GF) appeared in the ovary at $52 \mathrm{~h}$ later (AF) (Figure 1B). After injection of hCG, oocytes were discharged and CL formed in the ovary at $24 \mathrm{~h}$ later (Figure 1C); at $72 \mathrm{~h}$, ovaries contained many $\mathrm{CL}$ and a few degenerated/atresic follicles (DF) (data not shown).

In adult rat model, the ovaries on proestrus day (day $\mathrm{P}$ ) contained all kinds of follicles and some degenerating corpora lutea (DCL) (Figure 1D); and on estrus day (day E), the ovaries had newly formed CL (Figure 1E); on diestrus day (day D) ovaries mainly had CL.

In the aged rat model, ovaries of PE rats showed many follicular cysts that failed to ovulate (Figure 1F).

\section{Ovarian cGMP level and cGMP-PDE activity in the postnatal rat}

In postnatal rats, cGMP level was high on day of birth and decreased dramatically by Day 5, but increased to the highest level at Day 10 and then declined by Day 21. On the 
other hand, cGMP-PDE activity did not significantly change between Day 1 and 10, but increased significantly by Day 21 (Figure 2). There was no significant correlation between ovarian cGMP level and cGMP-PDE activity in this rat model.
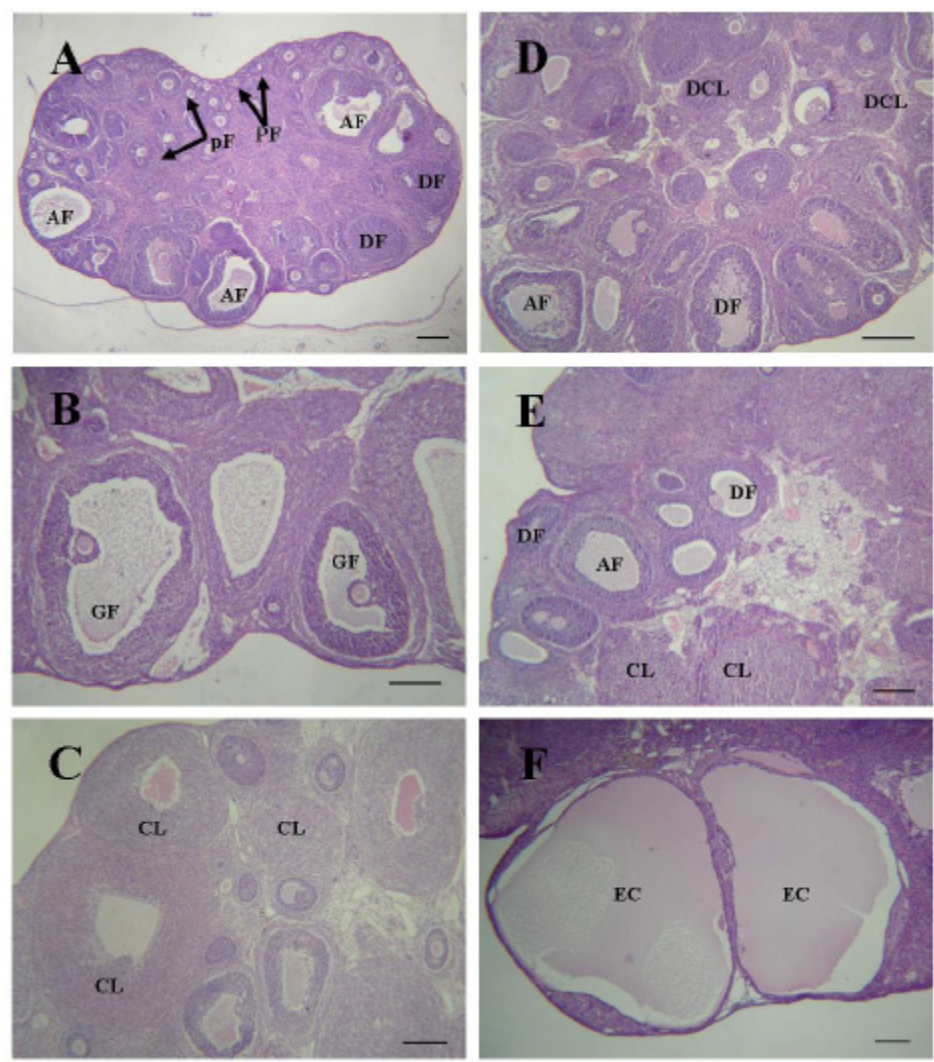

Figure 1. Morphologic examinations of ovaries in immature rats primed by gonadotropinsand adult rats during normal estrous cycles. A. A 25-day-old ovary containing different kinds of follicles, such as primordial follicles (PF), primary follicles $(\mathrm{pF})$, antral follicles (AF), and atresic follicles (DF), but not corpus lutea (CL). B. Preovulatory follicle (GF) appeared at the ovary at $52 \mathrm{~h}$ after injection of equine chorionic gonadotropin (eCG). C. Corpus luteal (CL) formed at the ovary at $24 \mathrm{~h}$ after injection of human chorionic gonadotropin (hCG). D) A ovary at proestrus day (P) of adult rats. E. A ovary at estrus day (E) of adult rats, containing corpus luteal (CL) formed in the ovary. F. A persistent estrous (PE) rat ovary containing established cystic follicles (EC). All sections were stained with hematoxylin and esosin (HE). Bar $=200 \mu \mathrm{m}$.

\section{Ovarian cGMP level and cGMP-PDE activity in immature rats primed by gonadotropin}

In immature female rats, cGMP level markedly decreased with eCG treatment, while cGMP-PDE activity did not show any significant changes. However, ovarian cGMP level and cGMP-PDE activity increased after injection of an ovulatory dose of hCG for induction of ovulation and luteinization (Figure 3). 

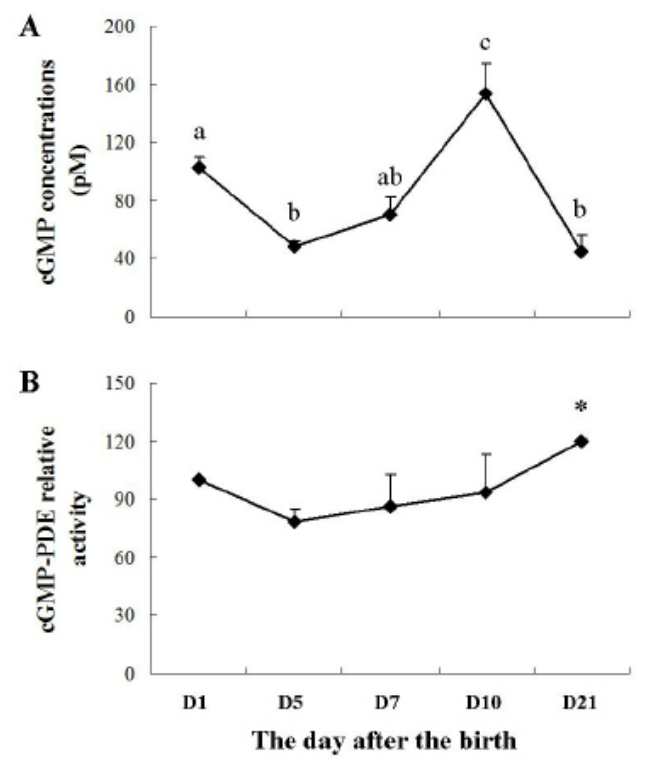

Figure 2. The concentration of cGMP (A) and relative activity of cGMP-PDE (B) in the ovarian homogenates of postnatal rats. Values represent as mean \pm SEM for 4 repeats. Different letters and star above the bars indicate a statistically significant difference among the days (Scheffe's post hoc test and paired or unpaired two-tailed Student's $t$-test).
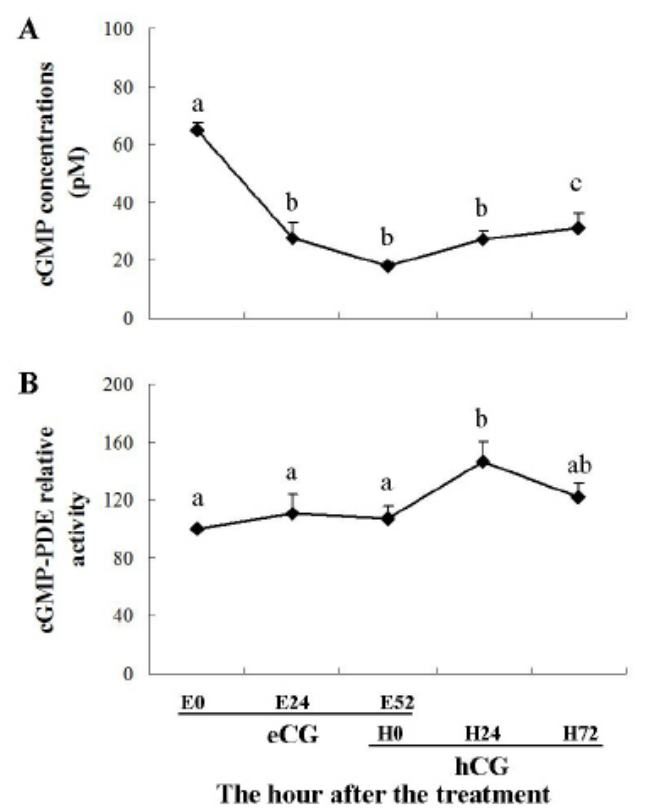

Figure 3. The concentration of cGMP (A) and relative activity of cGMP-PDE (B) in the ovarian homogenates of immature rats at $0,24,52 \mathrm{~h}$ after eCG (e0, e24, e 52) and 24, $72 \mathrm{~h}$ after $\mathrm{hCG}(\mathrm{h} 24, \mathrm{~h} 72)$. Values are the mean $\pm \mathrm{SEM}$ for 4 repeats. Different letters above the bars indicate a statistically significant difference among the treatments (Scheffe's post hoc test and paired or unpaired two-tailed Student's $t$-test). 


\section{Ovarian cGMP level and cGMP-PDE activity in adult rats}

In adult rats during normal estrous cycle, cGMP level was high on proestrus and metestrus days, while cGMP-PDE activity was only high on estrus day (Figure 4). There was a significant negative correlation between ovarian cGMP level and cGMP-PDE activity $(r=$ $-0.7715, \mathrm{~N}=16, \mathrm{P}<0.05)$ in this animal model.
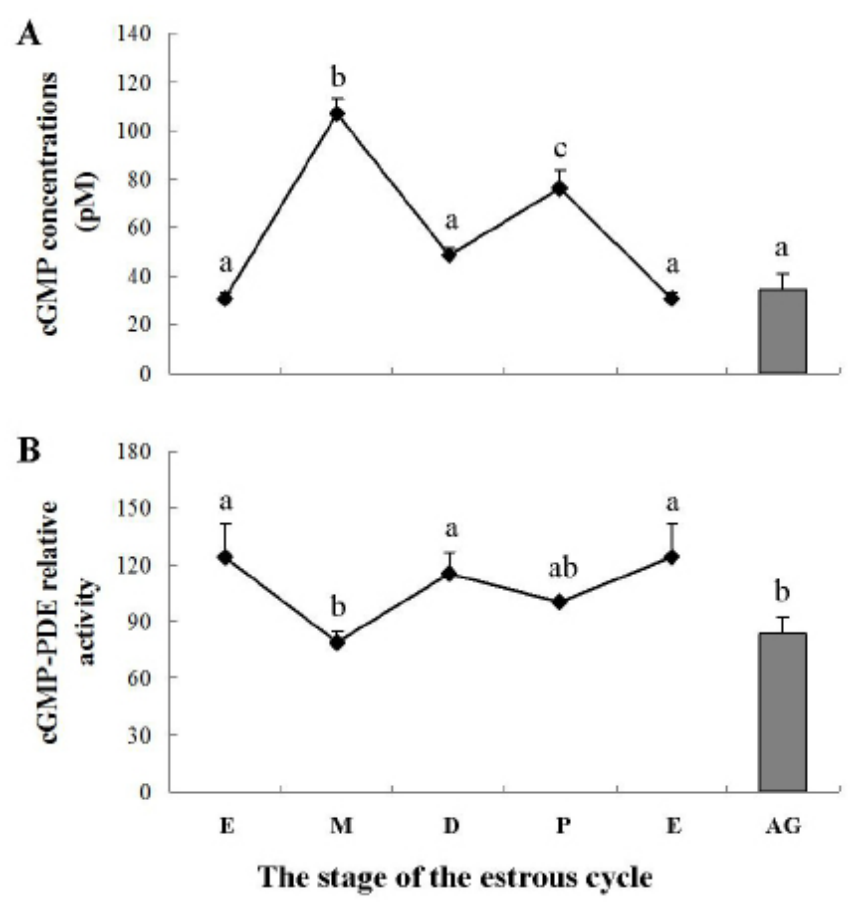

Figure 4. The concentration of cGMP (A) and relative activity of cGMP-PDE (B) in the ovarian homogenates of young adult rats at different estrous stages and PE rats. Values are the mean \pm SEM for 4 repeats. Different letters or star above the lines and bars indicate a statistically significant difference among the treatments (Scheffe's post hoc test and paired or unpaired two-tailed Student's $t$-test).

\section{Ovarian cGMP level and cGMP-PDE activity in acyclic aged PE rats}

In PE rats, ovarian cGMP level was similar to that in adult rats on estrus and diestrus days but lower than that on proestrus and metestrus days; ovarian cGMP-PDE activity was lower than that on estrus day but similar as on other estrous cycle days (Figure 4).

\section{DISCUSSION}

The present study clearly demonstrated that ovarian cGMP levels are not dependent on cGMP-PDE activity during early postnatal development, but are largely dependent on cGMP-PDE activity during estrous cycles in adult rats. This implies that the mechanisms of cGMP signaling involved in ovarian functions are stage-specific in the rat. 
Factors that control the initiation of primordial follicle development are one crucial aspect of ovarian biology and essential for female reproduction. Initiation of primordial follicle development determines the number of follicles available for dominant follicle selection and ovulation since females are born with a pool of oocytes that organize into primordial follicles, which represents the complete supply of oocytes that have the potential to be discharged (Shimizu, 2006). Therefore, we used the neonatal rat ovary model to examine the role of cGMP and cGMP-PDE in the initiation of ovarian follicle development. Morphological examinations were consistent with previous studies (Gelety and Magoffin, 1997; Braw-Tal, 2002; Shi and LaPolt, 2003), while analysis of cGMP levels revealed that cGMP signaling could participate in the initiation of the follicle growth, since an increase in cGMP level coincided with the development of the ovarian follicles accompanied by the development and proliferation of granulosa cells until 21 days. Our previous study revealed that the expression of sGC, which catalyzes the synthesis of cGMP, is mainly in the granulosa cells of primordial and preantral follicles, suggesting that cGMP signaling may be involved in the initiation of follicle growth. In addition to the well-recognized synthesis of cGMP, we also know that the unique way to breakdown cGMP is through hydrolysis catalyzed by specific PDE. Results of our current assay showed that during the early stage of follicle development, PDE activity did not significantly change before 10 days of age, suggesting that cGMP-PDE appears to have little effect on the pattern of early follicular growth and development. The dramatic changes in cGMP level and PDE activity on and after Day 21 are of greater interest to us, since these changes may be involved in the mechanisms regulating the ovarian functions via cGMP pathways, which dramatically change around Day 21 after birth in the rat.

In the gonadotropin-primed immature rats, synchronous recruitment and ovulation of a homogenous cohort of ovarian follicles are induced by the administration of exogenous gonadotropin on Day 25-26 to prepubertal female rats. In the present study, we found that ovarian cGMP levels decreased during follicular development after treatment with eCG, while cGMP-PDE activity changed little in this period, indicating the change in cGMP levels was mainly caused by decreased synthesis. This is consistent with the sGC expression pattern in our previous study (Shi et al., 2004). An increase in cGMP-PDE activity after hCG injection suggests that cGMP-PDE is the main regulator responsive to gonadotropin action, but the following decline may imply a complex mechanism of regulation in the ovary.

The estrous cycle, in fact, is a process of recruitment, development, selection, dominance, ovulation, and luteinization. In the present study, ovarian cGMP content decreased on day of estrus, while whole ovarian cAMP content increased in this period (Hubbard, 1983; Wang et al., 2007). These findings are consistent with the previous study of Hubbard, who found that these inverse changes in cyclic nucleotide concentrations were abolished when the preovulatory gonadotropin surges were blocked by phenobarbitone injection, but could be restored by administration of exogenous gonadotropins (Hubbard, 1983). The significant negative correlation between ovarian cGMP level and cGMP-PDE activity during the estrous cycle in the current study indicated that ovarian cGMP levels are largely dependent on ovarian cGMP-PDE activity in this adult rat model. Ovarian cGMP-PDE activity and cGMP concentration decreased in PE rats, which might have been due to the formation of follicular cysts. PE females display follicular growth, but fail to ovulate due to the absence of normal neuroendocrine function, resulting in the formation and accumulation of follicular cysts with degenerating granulosa layers (Shi and LaPolt, 2003). 
In conclusion, when our data are taken together with previous results, it appears that cGMP levels are mainly dependent on sGC expression and activity during early postnatal development, but subsequently dependent on cGMP-PDE activity largely after rats grow up. This implies that mechanisms of cGMP signaling involved in ovarian functions are stage specific in the rat. Further studies are required to elucidate the molecular mechanisms regulating cGMP-PDE enzyme expression and activity, the various influences of cGMP-PDE on ovarian functions, and interaction with other second messengers such as cAMP.

\section{ACKNOWLEDGMENTS}

Research supported in part by the National Natural Science Foundation of China (\#31101032, \#31271255), Ministry of Education Program for New Century Excellent Talents (\#NCET-12-0614), Doctoral Fund of Ministry of Education (\#20113503120002), Natural Science Foundation of Fujian Province (\#2011J01144) and Education Department of Fujian Province Science and Technology Project (\#AJ11041).

\section{REFERENCES}

Aharoni D, Dantes A, Oren M and Amsterdam A (1995). cAMP-mediated signals as determinants for apoptosis in primary granulosa cells. Exp. Cell Res. 218: 271-282.

Bradford MM (1976). A rapid and sensitive method for the quantitation of microgram quantities of protein utilizing the principle of protein-dye binding. Anal. Biochem. 72: 248-254.

Braw-Tal R (2002). The initiation of follicle growth: the oocyte or the somatic cells? Mol. Cell Endocrinol. 187: 11-18.

Broderick KE, MacPherson MR, Regulski M, Tully T, et al. (2003). Interactions between epithelial nitric oxide signaling and phosphodiesterase activity in Drosophila. Am. J. Physiol. Cell Physiol. 285: C1207-C1218.

Chaube SK, Prasad PV, Tripathi V and Shrivastav TG (2006). Clomiphene citrate inhibits gonadotropin-induced ovulation by reducing cyclic adenosine 3',5'-cyclic monophosphate and prostaglandin E2 levels in rat ovary. Fertil. Steril. 86: 1106-1111.

Das S, Maizels ET, DeManno D, St Clair E, et al. (1996). A stimulatory role of cyclic adenosine 3',5'-monophosphate in follicle-stimulating hormone-activated mitogen-activated protein kinase signaling pathway in rat ovarian granulosa cells. Endocrinology 137: 967-974.

Davies SA (2006). Signalling via cGMP: lessons from Drosophila. Cell Signal. 18: 409-421.

Gelety TJ and Magoffin DA (1997). Ontogeny of steroidogenic enzyme gene expression in ovarian theca-interstitial cells in the rat: regulation by a paracrine theca-differentiating factor prior to achieving luteinizing hormone responsiveness. Biol. Reprod. 56: 938-945.

Hubbard CJ (1983). The effect in vivo of alterations in gonadotropins and cyclic nucleotides on oocyte maturation in the hamster. Life Sci. 33: 1695-1702.

LaPolt PS, Leung K, Ishimaru R, Tafoya MA, et al. (2003). Roles of cyclic GMP in modulating ovarian functions. Reprod. Biomed. Online 6: 15-23.

Mullershausen F, Friebe A, Feil R, Thompson WJ, et al. (2003). Direct activation of PDE5 by cGMP: long-term effects within NO/cGMP signaling. J. Cell Biol. 160: 719-727.

Murthy KS (2001). Activation of phosphodiesterase 5 and inhibition of guanylate cyclase by cGMP-dependent protein kinase in smooth muscle. Biochem. J. 360: 199-208.

Nichols MR and Morimoto BH (2000). Differential inhibition of multiple cAMP phosphodiesterase isozymes by isoflavones and tyrphostins. Mol. Pharmacol. 57: 738-745.

Piriev NI, Yamashita CK, Shih J and Farber DB (2003). Expression of cone photoreceptor cGMP-phosphodiesterase alpha' subunit in Chinese hamster ovary, 293 human embryonic kidney, and Y79 retinoblastoma cells. Mol. Vis. 9: 80-86.

Richards JS, Fitzpatrick SL, Clemens JW, Morris JK, et al. (1995). Ovarian cell differentiation: a cascade of multiple hormones, cellular signals, and regulated genes. Recent Prog. Horm. Res. 50: 223-254.

Sette C and Conti M (1996). Phosphorylation and activation of a cAMP-specific phosphodiesterase by the cAMPdependent protein kinase. Involvement of serine 54 in the enzyme activation. J. Biol. Chem. 271: 16526-16534. 
Shi F and LaPolt PS (2003). Relationship between FoxO1 protein levels and follicular development, atresia, and luteinization in the rat ovary. J. Endocrinol. 179: 195-203.

Shi F, Stewart RL Jr, Perez E, Chen JY, et al. (2004). Cell-specific expression and regulation of soluble guanylyl cyclase alpha 1 and beta 1 subunits in the rat ovary. Biol. Reprod. 70: 1552-1561.

Shimizu T (2006). Promotion of ovarian follicular development by injecting vascular endothelial growth factor (VEGF) and growth differentiation factor 9 (GDF-9) genes. J. Reprod. Dev. 52: 23-32.

Sirotkin AV, Makarevich AV, Pivko J, Kotwica J, et al. (2000). Effect of cGMP analogues and protein kinase G blocker on secretory activity, apoptosis and the cAMP/protein kinase A system in porcine ovarian granulosa cells in vitro. $J$. Steroid Biochem. Mol. Biol. 74: 1-9.

Tatsukawa Y, Bowolaksono A, Nishimura R, Komiyama J, et al. (2006). Possible roles of intracellular cyclic AMP, protein kinase $\mathrm{C}$ and calcium ion in the apoptotic signaling pathway in bovine luteal cells. J. Reprod. Dev. 52: 517-522.

Theilig F, Bostanjoglo M, Pavenstadt H, Grupp C, et al. (2001). Cellular distribution and function of soluble guanylyl cyclase in rat kidney and liver. J. Am. Soc. Nephrol. 12: 2209-2220.

Wang Z and Shi F (2007). Phosphodiesterase 4 and compartmentalization of cyclic AMP signaling. Chin. Sci. Bull. 52: 34-46.

Wang Z, Huang R, Pan L and Li X (2006). Molecular structures, physiological roles and regulatory mechanisms of cyclic nucleotide-gated ion channels. Chin. J. Biochem. Mol. Biol. 22: 282-288.

Wang Z, Shi F, Jiang YQ, Lu LZ, et al. (2007). Changes of cyclic AMP levels and phosphodiesterase activities in the rat ovary. J. Reprod. Dev. 53: 717-725. 